\title{
EXCLUSIÓN E INCLUSIÓN EN LA IDUCACIÓN SUPERIOR: EL CASO DE LAS UNIVERSIDADES INTERCULTURALES EN MÉXICO*
}

Armando Alcántara**

\section{RESUMEN}

Al igual que en la mayoría de los países latinoamericanos, el acceso a la educación superior en México es todavía un privilegio. En la actualidad, sólo uno de cada cuatro jóvenes entre 19 y 23 años está inscrito en alguna institución de educación superior (IES). Esta proporción es aún menor al nivel estatal, ya que mientras los porcentajes de algunos estados del norte y del centro del país son considerablemente altos, los correspondientes a los estados del sur, caracterizados por sus altos niveles de población indígena, son muy bajos. Esta última población, representa un 10 por ciento del total de habitantes, la mayoría de los cuales viven en comunidades rurales y, muchas veces, aisladas. Si la sociedad mexicana fuera igualitaria, debería haber un 10 por ciento de estudiantes indígenas asistiendo a alguna IES, pero ello no es así. Una de las más recientes iniciativas del gobierno mexicano para enfrentar esta situación, ha sido la creación de las Universidades Interculturales, en aquellas comunidades con una población indígena considerable. Estas universidades tienen como objetivo principal la formación de profesionales e intelectuales comprometidos con el desarrollo de sus comunidades y regiones. En el centro de su misión académica está el estudio de sus respectivas culturas y lenguas. Este artículo examina la situación actual de este tipo de instituciones y analiza algunas de sus contribuciones al reconocimiento de las culturas indígenas y locales, así como su papel como instrumentos de justicia social.

Palavras-chave: universidades intercultruales; acesso-inclusión; educación superior.

\footnotetext{
* Artigo recebido em 15/03/2008 e aprovado 3/05/2008.

** Universidad Nacional Autónoma de México. E-mail: aralsan@servidor. unam.mx
} 


\section{INTRODUCCIÓN}

Las universidades y demás instituciones de educación superior actualmente, están enfrentando un cambio sin precedentes: de ser pequeñas instituciones al servicio de las elites, se han transformado en instituciones de masas que educan a miles de estudiantes de diferentes condiciones socioculturales. Durante los últimos años los estudios y debates llevados a cabo en diferentes países, han llegado a la conclusión de que sin más y mejor educación superior, los países en desarrollo tendrán mayores dificultades para beneficiarse de la economía global basada en el conocimiento (Task Force, 2000). A pesar del impresionante crecimiento del número de estudiantes universitarios--particularmente en el llamado mundo en desarrollo--la calidad se ha ido deteriorando debido a la falta de recursos financieros, y la inadecuada preparación de los docentes. El carácter masivo de la educación superior, los beneficios que otorga a los individuos y a la sociedad, la han hecho muy atractiva para todos los grupos y sectores.

La diversidad cultural es una característica relevante de las sociedades de América Latina y del Caribe. La herencia cultural de estas sociedades es, principalmente, el resultado de la combinación de componentes indígenas, africanos y europeos, aunque la presencia de Asia también puede advertirse en países como Perú y Brasil. Centurias de colonización europea (siglos XVI a XIX), produjeron explotación y depredación para las poblaciones de ascendencia indígena y africana en la región, pese a la proclamación de independencia en la mayoría de los países durante los siglos XVIII y XIX. El pleno reconocimiento a las culturas de origen indígena y africano, en la mayoría de los países latinoamericanos y del Caribe, sólo se ha dado hasta años recientes.

El derecho a la educación superior, dentro de este cambiante contexto, constituye uno de los factores indispensables en la adquisición de la ciudadanía plena y la justicia social para todos los sectores de la sociedad. En este sentido, y de acuerdo con el Artículo 26.1 de la Declaración Universal de los Derechos Humanos la admisión a la educación superior debe basarse en el mérito, la capacidad, el esfuerzo, la perseverancia y la dedicación demostrada por quienes buscan tener acceso a ella. Por otra parte y, desde la perspectiva del aprendizaje a lo largo de toda la vida, la educación superior puede tener lugar a cualquier edad, con el debido reconocimiento de las habilidades y los conocimientos 
previamente adquiridos. Por lo tanto, en el acceso a la enseñanza superior la discriminación no puede ser aceptada bajo ninguna circunstancia por causa de raza, sexo, idioma, religión o distinción económica, social o cultural, ni por limitaciones físicas.

Asimismo, el Artículo 3..$^{\circ}$ de la Declaración Mundial sobre Educación Superior para el Siglo XXI establece que

Debe facilitarse el acceso a la educación superior a los integrantes de grupos desfavorecidos, tales como los pueblos indígenas, las minorías culturales y lingüísticas, pueblos que viven bajo la ocupación extranjera, así como a quienes tienen limitaciones físicas; puesto que estos grupos tanto de manera colectiva como individual poseen el talento y la experiencia que es de gran valor para el desarrollo de las sociedades y las naciones. Algunas soluciones en forma de ayuda material y educacional, podrían contribuir a superar los obstáculos que enfrentan dichos grupos, tanto para acceder como para mantenerse en la educación superior (UNESCO, 1998, p.6).

Algunos de los puntos principales que se discuten en este trabajo tienen que ver con las demandas de los pueblos indígenas para acceder a la educación superior en México, y las características de las instituciones que se han creado para este propósito. Por ello, la primera parte del mismo, describe la condición social de los pueblos indígenas de América Latina y el Caribe. La segunda parte explora varias de las causas por las cuales dichos pueblos han sido excluidos del acceso a la educación superior. Mientras que la tercera parte examina algunas de las políticas e iniciativas que los gobiernos latinoamericanos han puesto en marcha, para responder a las demandas de las poblaciones indígenas de contar con mejores oportunidades educativas en el nivel superior. La cuarta parte analiza el caso de México, particularmente la situación de sus pueblos indígenas y los obstáculos que enfrentan en la búsqueda de posibilidades más amplias de educación en el nivel universitario. Los principios fundamentales de las universidades culturales se describen en la quinta parte. En el siguiente apartado se presenta el caso de la Universidad Intercultural del Estado de México (UIEM), una de las instituciones de educación superior recientemente creadas, esta parte también incluye un esbozo de los programas de licenciatura de la UIEM. La séptima parte de este trabajo discute de manera amplia los términos intercultural e indígena, aplicados en las nuevas universidades creadas para satisfacer las 
demandas de los pueblos indígenas. El octavo apartado incluye una serie de desafíos que enfrentan las universidades interculturales en el presente y en el futuro inmediato. Finalmente, hay una sección de conclusiones, e implicaciones de política sobre el contenido antes descrito.

\section{EDUCACIÓN SUPERIOR Y PUEBLOS INDÍGENAS EN AMÉRICA LATINA}

En años recientes los pueblos indígenas se han convertido en los actores principales de las arenas políticas y culturales en su lucha por mayor acceso a la educación superior. Algunos de estos movimientos tuvieron su culminación en la década de los noventa y los primeros años del presente siglo, pero algunas otras están todavía en desarrollo. La iniciativa de las Naciones Unidas para declarar el período 1994-2004 como la Década de los Pueblos Indígenas contribuyó a reforzar dichas demandas, quedando claro que eran necesarias esas medidas a fin de reducir las enormes asimetrías derivadas de la exclusión, la invisibilidad y la negación de la existencia de esos pueblos.

Un nuevo concepto de "desarrollo con identidad" actualmente en proceso de consolidación, reconoce las condiciones de pobreza material, desigualdad y exclusión de las poblaciones indígenas así como el potencial de sus bienes culturales, naturales y sociales. Este reconocimiento busca ampliar las oportunidades - con equidad de género - de desarrollo socioeconómico y, al mismo tiempo, reforzar su identidad, cultura, territorialidad, recursos naturales y organización social, bajo la premisa de que el desarrollo sustentable requiere de la iniciativa y empowerment de sus beneficiarios, del respeto de sus derechos individuales y colectivos y el reconocimiento de que el desarrollo de los pueblos indígenas beneficia de manera significativa a la sociedad en general (Muñoz, 2006)

Aún a comienzos del siglo XXI los pueblos originarios de América Latina continúan padeciendo la exclusión social mediante diferentes formas de negación e invisibilidad: judicial, socioeconómica, cultural y educativa. No se debe olvidar que las identidades nacionales de los países de la región se construyeron, en gran parte, sobre la base de negar su diversidad así como sus raíces culturales y étnicas. Dentro del marco de tales estados-nación, el concepto de ciudadanía sólo incluía a quieres eran hombres, blancos, propietarios y heterosexuales. Esta restringida concepción de lo que era ser ciudadano, se plasmó en las constituciones de la mayoría de los estados latinoamericanos, creándose así una cultura 
política discriminatoria y excluyente. ${ }^{1}$ Por lo que respecta a la educación superior, no existe en nuestros días ninguna legislación que contemple la participación de los pueblos indígenas en los sistemas educativos de dicho nivel.

La población indígena en América Latina varía entre 40 y 50 millones, cerca del 10 por ciento de la población total de la región. Sin embargo, la condición económica de los pueblos indígenas, en cualquiera de los países de esta área geográfica, significa ser pobre entre los pobres. De acuerdo con estudios realizados por el Banco Mundial (BM) y el Banco Interamericano de Desarrollo (BID), el nivel de ingresos y otros indicadores del desarrollo humano de los habitantes de las zonas indígenas, muestran que éstos permanecen en el fondo de la escala económica. Esto ocurre en un contexto de fuerte desigualdad donde el 10 por ciento de los individuos más ricos acapara entre el 40 y el 47 por ciento del ingreso total, mientras que al 20 por ciento más pobre le corresponde solamente entre el 2 y el 4 por ciento. México ocupa el quinto lugar entre las sociedades más desiguales de América Latina, después de Brasil, Guatemala, Colombia y Chile. Del mismo modo, la brecha educativa entre los habitantes de poblaciones indígenas y no indígenas en la región es de 3.7 años en Bolivia, 2.6 en Ecuador, 3.2 en Guatemala y 3.3 en México (Muñoz, 2006).

CAUSAS DE LA EXCLUSIÓN DE LOS INDÍGENAS EN LA EDUCACIÓN SUPERIOR

Algunas de las causas que explican dicha exclusión son las siguientes: la dispersión geográfica de las comunidades y falta de instituciones educativas localizadas en áreas con población indígena; la alta diversidad lingüística y ausencia de especialistas en esas lenguas; las poblaciones indígenas se localizan en zonas marginadas, con grandes carencias y altamente conflictivas, haciendo difícil la creación de instituciones educativas; el currículum de las universidades es, con frecuencia, irrelevante para el tipo de trabajos que prevalecen en las comunidades indígenas; la discriminación en los mercados laborales urbanos para profesionales con una experiencia en asuntos indígenas; los bajos niveles de calidad y cobertura de la educación básica y media superior para los pueblos indígenas, determinan la escasez de potenciales estudiantes universitarios; existen altas tasas de deserción entre los estudiantes de origen indígena, 
debido a las grandes distancias entre las comunidades indígenas y las escuelas, así como a las diferencias culturales y los altos costos que representan para las familias el enviar a sus hijos a escuelas ubicadas fuera de sus comunidades; la falta de políticas públicas orientadas a superar las barreras y restricciones al acceso; la carencia de instituciones de educación superior para los pueblos indígenas; los estudiantes indígenas consideran que la enseñanza superior debería brindarles conocimientos prácticos y habilidades específicas, en lugar de conocimientos generales que pueden ser de poca o ninguna utilidad en sus comunidades; las universidades tradicionales carecen de la suficiente flexibilidad para admitir estudiantes provenientes de diferentes culturas; las universidades tienen currícula con poco contenido relevante para las comunidades indígenas, y la educación universitaria se convierte en algo abstracto al no formar estudiantes con habilidades y destrezas específicas; el aprendizaje en las comunidades indígenas es una experiencia colectiva, en tanto que el modelo de aprendizaje predominante, en la mayoría de las universidades, es individualista y altamente competitivo.

Por lo tanto, además de la falta de recursos económicos, sociales y culturales para sobrevivir dentro del sistema educativo, los pueblos indígenas también enfrentan enormes obstáculos dentro de los establecimientos universitarios, impidiéndoles contar con las suficientes y relevantes oportunidades de alcanzar los más altos niveles de aprendizaje.

PResenCIA DE LOS PUEBLOS INDÍGENAS EN LA EDUCACIÓN SUPERIOR DE América Latina

La década de los noventa en América Latina fue testigo de varias luchas y demandas de los pueblos indígenas para lograr el reconocimiento social y cultural dentro de sus respectivas sociedades. Hubo casos en los cuales dichos movimientos y demandas tomaron la forma de luchas de resistencia, algunas pacíficas y otras - como la de los Zapatistas en México - fueron armadas. ${ }^{2}$ Uno de los propósitos principales de estos movimientos fue demandar el reconocimiento de sus prácticas culturales - idioma, identidad y costumbres -, dentro de las sociedades latinoamericanas. De esta forma los pueblos indígenas se convirtieron en importantes actores dentro de la escena política - a nivel nacional e internacional - dejando atrás décadas de servidumbre y explotación. Con el objeto de responder a las demandas de las poblaciones indígenas 
por mayor acceso a la educación superior, algunos de los gobiernos latinoamericanos han puesto en marcha una serie de políticas educativas que incluyen el otorgamiento de becas, cuotas en la matrícula, admisión especial, programas universitarios específicos, modalidades de educación no formal, cursos de preparación para ingresar a la universidad, acceso a la educación virtual internacional y la creación de universidades indígenas o interculturales.

Los programas de becas consisten en incentivos económicos proporcionados directamente a los estudiantes de comunidades específicas. Las becas se consideran instrumentos para mantener a los estudiantes en la escuela, evitando su deserción por razones económicas. El programa de becas en México se denomina PRONABES (Programa Nacional de Becas para la Educación Superior).

Las cuotas en la matrícula pueden considerarse una especie de programas de acción afirmativa. Las universidades determinan un cierto porcentaje de admisiones para estudiantes de comunidades indígenas que no podrían ser admitidos mediante los procedimientos normales. Estos programas han provocado algunas controversias y debates en los que algunas personas argumentan que el dar facilidades a los estudiantes de grupos en desventaja trae como consecuencia una especie de "etiqueta" de bajo rendimiento o preferencia especial. En otras palabras esos estudiantes podrían ser criticados porque su admisión no se basó en sus méritos académicos sino en la acción afirmativa.

Las admisiones especiales son similares a las del punto anterior, porque resultan de acuerdos especiales entre las universidades y los miembros de las comunidades indígenas. Se dan casos en los que estudiantes admitidos mediante estos programas son familiares de profesores o de empleados de las universidades.

Los programas universitarios incluyen acuerdos entre las comunidades indígenas y las universidades para establecer programas o cursos específicos destinados a satisfacer necesidades específicas.

Las modalidades de educación no formal consisten en la realización de actividades académicas extracurriculares. Dichas actividades pueden ser desarrolladas por ONGs, universidades u organismos gubernamentales.

Los cursos de preparación para ingresar a la universidad tienen por objetivo mejorar el nivel académico de los estudiantes antes de la realización de los procedimientos de admisión a los programas universitarios. 
El acceso a la educación virtual internacional incluye políticas orientadas a promover la conectividad en lugares apartados de las principales ciudades mediante la educación a distancia.

La creación de universidades indígenas o interculturales pueden ser promovidas por iniciativas públicas, privadas o de las propias comunidades. Dichas instituciones buscan satisfacer las necesidades específicas de educación superior de los grupos indígenas, construyendo sus propias currícula junto con la creación de mecanismos particulares de admisión, diferentes del resto de las universidades públicas y privadas (Muñoz, 2006).

LOS PUEBLOS INDÍGENAS EN EL SISTEMA MEXICANO DE EDUCACIÓN SUPERIOR

Como en la mayoría de los países de América Latina y el Caribe, así como en el resto del mundo en desarrollo, el acceso a la educación superior en México es todavía un privilegio. Sólo uno de cada cinco jóvenes de 19 a 23 años asiste a alguna institución de educación superior, ${ }^{3}$ si bien a principios de los años noventa la proporción era de 12 por cada 100 en las instituciones públicas y privadas. La educación superior pública ha mostrado un claro proceso de masificación. Sin embargo, dicho porcentaje es relativo en un país que como México tiene enormes desigualdades. Las cifras son muy diferentes, por ejemplo en la Ciudad de México y en los estados más pobres como Oaxaca, Guerrero y Chiapas. Las oportunidades para un joven en la capital del país cuadruplican las de un joven habitante de alguno de los estados mencionados (Schmelkes, 2003).

Con pocas excepciones, la mayor parte de las aproximadamente 2000 instituciones de educación superior se localiza en áreas urbanas. El acceso para los sectores rurales e indígenas de la población resulta significativamente oneroso para las familias. Esta es una de las causas del reducido número de estudiantes de esos sectores en las universidades. Pero existe otro factor, tal vez más importante que el económico: la calidad de la educación que los estudiantes de estas poblaciones reciben en los niveles previos a la educación superior. De acuerdo con diversas evaluaciones llevadas a cabo en todo el país, la calidad de la educación en México está segmentada. Aquellos con las mayores desventajas son estudiantes que asisten a escuelas ubicadas en áreas rurales e indígenas. Los egresados de dichas instituciones raras veces aprueban los exámenes 
de admisión en universidades y otros establecimientos de enseñanza superior. Schmelkes (2003) señala que pese a la falta de datos relacionados con el número de campesinos e indígenas, se estima que sólo representan el uno por ciento de los estudiantes universitarios, y la mayoría de ellos son indígenas cuyos padres migraron a las áreas urbanas, que asistieron a escuelas públicas en tales áreas y cuya eficiencia y calidad era significativamente mejor que la de las escuelas localizadas en zonas rurales $\mathrm{o}$ indígenas.

Casi la mitad de los estudiantes indígenas que asisten a la universidad en México, representa la primera generación de su familia que lo hace. Éste es un claro indicador de la enorme inequidad educativa que han padecido hasta hoy. Más aún, probablemente uno de los principales factores de las diferencias en la calidad educativa mencionados en el párrafo anterior, es la falta de relevancia cultural y lingüística del currículum de las escuelas en las poblaciones indígenas. Se estima que la población indígena representa el 10 por ciento del total de habitantes del país. Si el sistema educativo mexicano fuera justo, debería haber 10 por ciento de indígenas en la educación superior. Dado que no es así, se trata más bien de un indicador de racismo estructural y de inequidad educativa enraizada en el sistema de educación.

Con el fin de incrementar la participación de los pueblos indígenas en el sistema de educación superior, desde el comienzo del presente siglo, el gobierno mexicano estableció la meta de triplicar su presencia en dicho nivel. Para lograr esa meta se implementaron tres estrategias. La primera fue el sistema de becas llamado PRONABES. La cantidad de becas otorgadas a los estudiantes de estas poblaciones no ha sido muy significativa debido a que, como ya se mencionó, existe sólo un pequeño número de esos estudiantes en las instituciones de educación superior. La segunda estrategia ha sido la creación de las llamadas universidades interculturales establecidas en áreas en donde predomina la población indígena. La tercera estrategia combina un programa de apoyo económico (becas o crédito educativo) con una transformación de las instituciones receptoras. Dichas transformaciones incluyen la creación de cursos especiales encaminados a nivelar su formación académica previa, tutorías, programas de orientación académica, "adopción" de estudiantes indígenas por parte de profesores y compañeros de clase. Puesto que las universidades interculturales constituyen una de las más promisorias e 
innovadoras iniciativas para proveer acceso a la educación superior a los pueblos indígenas, ese será el tema del siguiente apartado.

\section{LAS UNIVERSIDADES INTERCULTURALES EN MÉXICO}

Estas instituciones han definido de la siguiente forma sus principios fundamentales:

1) Misión institucional: la formación de profesionales e intelectuales comprometidos con el desarrollo de sus pueblos y regiones. Las universidades interculturales deberán ofrecer educación de calidad. Los graduados de estas instituciones serán capaces de continuar estudios de posgrado u obtener un empleo en cualquier lugar. El principal interés de estas universidades será promover el desarrollo de la región, buscando que los graduados permanezcan o regresen a su propia región.

2) La investigación acerca de las culturas y los idiomas de las comunidades indígenas de los alrededores de las universidades interculturales, está en el centro de cada institución. La docencia y la extensión también se orientan a servir a esas comunidades.

3) Los programas educativos se derivan de las necesidades y el desarrollo potencial de cada región. En consecuencia, el currículo tiene una orientación flexible. Los estudiantes matriculados en los programas de licenciatura pueden obtener un diploma de profesional asociado, después de haber completado dos años de estudios. Los programas académicos son dinámicos y susceptibles de ser actualizados constantemente, sin saturar los mercados laborales regionales. Siendo conscientes que con frecuencia los mercados laborales tradicionales son débiles o inexistentes, estos programas forman a los estudiantes en destrezas y habilidades para el auto empleo o el empleo colectivo.

4) La admisión de los estudiantes no se basa en los criterios académicos tradicionales, porque se reconoce que la calidad de la formación previa de la mayoría de los aspirantes es muy baja. En lugar de seleccionar sólo a los mejores candidatos, la estrategia aspira a elevar su nivel académico durante el primer año. El énfasis está puesto en la lengua materna de los estudiantes, español, inglés, matemáticas y computación, así como en el desarrollo y fortalecimiento de las habilidades del pensamiento superior y el proceso de aprender a aprender. 
El proceso de admisión a las Universidades Interculturales es un asunto crítico, de acuerdo con el Observatorio Ciudadano de la Educación (OCE, 2004). Este grupo de especialistas en educación considera que la forma en la cual las nuevas universidades van a resolver el complicado problema de las admisiones no está suficientemente claro. A este respecto han planteado los siguientes cuestionamientos:

a) Si las Universidades Interculturales son "instituciones abiertas", ¿la estrategia de admisión se debería aplicar equitativamente a quienes pertenecen a las poblaciones indígenas y a quienes no? El punto es como asegurarse que los estudiantes de nuevo ingreso son capaces de llevar a cabo sus programas académicos y completarlos de manera exitosa.

b) Si por razones de justicia se da libre acceso, sin ninguna otra condición que la solicitud, ¿no se estarían debilitando los criterios académicos y creando, finalmente, una mayor inequidad?

c) ¿El pensar acerca de condiciones justas para ingresar a estas universidades no debería ser una buena oportunidad para desarrollar formas más creativas de realizar la selección académica sin causar mayor exclusión?

Reconocen, sin embargo, que las Universidades Interculturales son instituciones nuevas, y que algunas de las implicaciones de política, y los cuestionamientos anteriores, deberían ser respondidas para el fortalecimiento de la vida institucional.

5) Las Universidades Interculturales están estrechamente vinculadas a las comunidades indígenas de su entorno, mediante la construcción e implementación de un proyecto de desarrollo (Schmelkes, 2003).

\section{La Universidad Intercultural del Estado de MéXico (UIEM)}

Actualmente existen en México alrededor de 10 universidades interculturales, ya sea en funcionamiento o en proceso de terminación. La Universidad Intercultural del Estado de México (UIEM) es un ejemplo de ellas y puede tomarse como ejemplo para describir sus objetivos y propósito así como su currículo. La UIEM se inauguró en septiembre de 2004 con el propósito principal de satisfacer la demanda social de educación superior con un nuevo enfoque educativo, centrado principalmente, pero no sólo, en los grupos indígenas. La UIEM acepta estu- 
diantes de todos los orígenes culturales y étnicos. Uno de los principios fundamentales de esta nueva institución es cumplir el ofrecimiento de otorgar la educación a los indigenas en sus propias lenguas. En la misión de la universidad se establece que los profesionales e intelectuales formados en ella, deberán realizar tareas de promoción de actividades que favorezcan el proceso de revaloración y revitalización de las lenguas y culturas indígenas. También se busca tener una activa participación en el desarrollo sustentable de la región y una profunda interacción social dentro del marco de la diversificación cultural. La primera generación de la UIEM estuvo formada por 271 estudiantes entre 17 y 23 años de edad, de los cuales el 70 por ciento pertenece a alguno de los pueblos indígenas, siendo el 60 por ciento mujeres. Los estudiantes provenían de distintos municipios pertenecientes a ocho estados diferentes, y la mitad del profesorado es bilingüe.

Los primeros tres programas de licenciatura ofrecidos por esta nueva institución fueron los siguientes:

1) Lengua y cultura, cuyo principal objetivo consiste en desarrollar habilidades comunicativas y favorecer el dominio de una segunda lengua, manteniendo así la filosofía de las culturas originarias de preservar el uso de una lengua indígenas, y establecer una amplia comunicación mediante el respeto de las diferencias con otras culturas de México y del resto del mundo. Se espera que los graduados de este programa sean capaces de desempeñarse como traductores e intérpretes. Podrán trabajar en instituciones de educación media y superior, empresas editoriales, radiodifusoras y organizaciones no gubernamentales (ONGs).

2) Comunicación intercultural, el propósito de este programa es formar cuadros de expertos capaces de manejar procesos comunicacionales, usando principalmente lenguas indígenas. Este programa también incluye una formación especial en el diseño, manejo, administración y gestión de proyectos comunitarios relacionados con la comunicación impresa y el procesamiento de noticias, diseño gráfico, producción de radio, locución, etcétera. Los graduados desarrollarán cualquiera de estas actividades en proyectos de comunicación comunitarios.

3) Desarrollo sustentable, cuyo principal objetivo es la promoción de modelos de producción realizados en armonía con los ambientes natural y cultural, así como el desarrollo económico a nivel nacional, estatal, regional y local, en permanente vinculación con propuestas 
alternativas de desarrollo. Los graduados de este programa habrán de promover y fortalecer las economías locales dentro del marco de respeto a la diversidad ambiental y cultura, teniendo también capacidad de tomar decisiones basadas en un conocimiento holístico (conocimiento científico y conocimiento indígena tradicional). Los graduados podrían laborar en el desarrollo de ecotecnologías, diseño e instrumentación de proyectos de desarrollo sustentable, empresas públicas y privadas, organizaciones civiles, ONGs relacionadas con la producción sustentable y centros de investigación (UIEM, página electrónica).

\section{¿UNIVERSIDADES INDÍGENAS O INTERCULTURALES?}

La interculturalidad ha sido conceptualizada en forma amplia como una relación de respeto para el enriquecimiento mutuo entre culturas. Es por esto que la educación intercultural no puede restringirse sólo a las comunidades indígenas. Por el contrario, tiene que alcanzar a toda la población. Dentro de los pueblos indígenas, la educación intercultural busca tener un mejor conocimiento y una apreciación más justa de la cultura propia, así como el reconocimiento del valor y el respeto a las otras culturas con las que se comparte el territorio. Reconocer el valor de esta contribución y el consecuente respeto y apreciación por los diferentes pueblos y grupos culturales es otro de los objetivos de esta conceptualización. La sociedad mexicana debería valorar y apreciar la diversidad cultural y considerar un privilegio el hecho de vivir en un país pluriétnico y multicultural, y hallar en ello una fuente esencial de enriquecimiento personal y cultural. Al hacerlo será posible eliminar actitudes y conductas que impliquen prejuicio, discriminación y racismo, las cuales han tenido, por desgracia, una fuerte presencia en las concepciones y el proceder cotidiano de una parte importante de los mexicanos (Schmelkes, 2001).

El politólogo venezolano Daniel Mato considera que, como consecuencia de la importancia creciente de iniciativas y políticas recientes, comúnmente denominadas "interculturales", orientadas al reconocimiento y manejo de la diversidad cultural de formas equitativas y mutuamente respetuosas, se ha extendido la idea de que la "interculturalidad" se refiere de manera exclusiva a objetivos o experiencias que procuran reconocimiento y respeto mutuo entre grupos humanos culturalmente diversos. No obstante, señala Mato (2007), un uso más cuidadoso del 
término "interculturalidad" hace necesario incluir en este concepto todo tipo de experiencias humanas caracterizadas por la relevancia de dinámicas de relación entre grupos humanos que se perciben mutuamente como poseedores de una diversidad cultural. En consecuencia, la idea de interculturalidad puede referirse a diferencias étnicas, religiosas, socioeconómicas e identitarias.

\section{DESAFÍOS PARA LAS UNIVERSIDADES INTERCULTURALES}

Con el fin de alcanzar su mayor potencial como instituciones, las universidades interculturales tendrán que superar una serie de obstáculos y retos. Primero será el asunto de la relevancia, para lo cual necesitarán establecer mecanismos para revisar de manera continua y actualizar sus programas académicos a fin de poder integrar sus intereses con los de las comunidades que las rodean. En segundo lugar, está el tema de la política de admisiones, tal como fue planteada por el Observatorio Ciudadano de la Educación. El dilema será cómo conciliar las legítimas demandas de los pueblos indígenas con otros grupos, para tener acceso a la educación superior sin debilitar la calidad de los estándares académicos. En este sentido, la justicia social no deberá entrar en conflicto con la calidad de la educación que se ofrece. En tercer término, se encuentra el desafío de evitar el aislamiento institucional. Las universidades interculturales deberán permanecer en contacto permanente con las comunidades aledañas y la sociedad en general, a fin de que no se conviertan en ghettos culturales. En este orden de cosas, el concepto de interculturalidad implica un rasgo más inclusivo para las nuevas universidades. En cuarto lugar, está el asunto financiero, el cual constituye en la actualidad un problema común para todas las universidades públicas mexicanas. Tendrán que explorar nuevas y efectivas estrategias para obtener recursos adicionales al presupuesto oficial, sin comprometer su libertad académica.

\section{Conclusiones}

Las universidades interculturales constituyen instituciones innovadoras y son instrumentos de justicia social para ampliar el acceso a la educación superior de grupos desfavorecidos o abandonados, principalmente - pero no exclusivamente - indígenas. La creación de estas 
nuevas instituciones ha sido el producto de prolongadas luchas llevadas a cabo durante décadas en diversos países de América Latina y el Caribe. Las universidades interculturales celebran y ponen en el centro de sus obligaciones académicas, la diversidad cultural y lingüística que caracteriza a las sociedades de la región. En algunos casos, los gobiernos fueron lo suficientemente sensibles para reconocer y dar la bienvenida a las demandas de estos grupos, y por tanto promovieron activamente la creación de dichas universidades. En otros casos, fueron los mismos grupos quienes llevaron adelante sus iniciativas y crearon sus propias instituciones comunitarias.

En tanto instituciones nuevas, a veces frágiles y en pleno proceso de consolidación, las universidades interculturales enfrentan enormes desafíos y riesgos, tales como la relevancia, la calidad de la educación, la integración y el aislamiento, así como el financiamiento, entre otros. La permanencia y el fortalecimiento institucional de las universidades interculturales requieren, entonces, de la activa participación de las comunidades indígenas, el gobierno (en los niveles nacional, estatal, regional y local) y las propias comunidades académicas.

\section{ABSTRACT}

As in other Latin American countries, access to higher education in Mexico is still a privilege. Currently, just one in four 19 to 23 -year-olds is enrolled at an institute of higher education (HE). This average is even lower at State level: while averages in some northern and central states are considerably high, those in the southern states, with a significant indigenous population, are very low. Indigenous people, who live in rural and sometimes isolated communities, represent 10 per cent of Mexico's total population. If Mexican society were equalitarian, 10 per cent of indigenous students would be attending some form of higher education, but this is not so. One of the most recent strategies carried out by the Mexican government has been the creation of Intercultural Universities in communities with a significant indigenous population. These universities set out to train professionals and intellectuals committed to the development of their communities and regions. At the center of their academic mission is the study of their respective culture and language. This paper will examine the current situation of intercultural universities in Mexico and will also assess their contribution to acknowledging indigenous and local cultures as well as their role in the search for social justice.

Keywords: Intercultural universities. Access-inclusion. Higher education. 


\section{NOTAS}

1. El libro de Carlos Alberto Torres Democracia, educación y multiculturlismo. Dilemas de la ciudadanía en un mundo global, es un análisis profundo e interesante de estos temas.

2. Vale la pena hacer notar la "coincidencia" ocurrida en México al inicio de 1994. Por un lado, el presidente Carlos Salinas (1988-1994) firmó el Tratado de Libre Comercio con América del Norte (TLCAN o NAFTA) con los Estados Unidos y Canadá. Salinas celebró la firma del acuerdo como la entrada de México al Primer Mundo. Por el otro lado, ese mismo día el levantamiento Zapatista en Chiapas, significó un fuerte rechazo del sector más pobre de la sociedad mexicana a la estrategia económica seguida por el gobierno, la cual había comenzado a principio de los ochenta, y que produjo una enorme concentración de la riqueza en manos de unos cuantos, y niveles sin precedente de pobreza para la mayoría de la población. En el centro de la protesta armada estaba también la determinación de poner fin a la invisibilidad e injusticia que por siglos ha sufrido la población indígena.

3. Datos más recientes del gobierno mexicano indican que uno de cada cuatro jóvenes de 19 a 23 años está matriculado en alguna de las diferentes instituciones de educación superior (Poder Ejecutivo Federal, 2007).

\section{REFERENCIAS}

MATO, D. Interculturalidad y Educación Superior en América Latina y el Caribe de UNESCO-IESALC. Boletín Digital IESALC-UNESCO, 137, Junio, 2007.

MUÑOZ, M. R. Educación Superior y Pueblos Indígenas en América Latina y el Caribe. En Informe sobre la Educación Superior en América Latina y el Caribe 2000-2005. IESALC-UNESCO. Caracas: IESALC-UNESCO; pp. 129-142, 2006.

OBSERVATORIO CIUDADANO DE LA EDUCACIÓN (OCE). Comunicado 122 Universidades Interculturales. México DF. OCE, 2004.

PODER EJECUTIVO FEDERAL. Plan Nacional de Desarrollo 2007-2012. México DF: Poder Ejecutivo Federal, 2007

SCHMELKES, S. México: Educación Intercultural Bilingüe Destinada a los Pueblos Indígenas. Texto base del V Foro Virtual Educación para la Interculturalidad, Migración-Desplazamiento y Derechos Lingüísticos organizado por el Foro Latinoamericano de Políticas Educativas (FLAPE). (http://www. aulaintercultural.org) Agosto 29, 2007. 
Educación Intercultural. El Caso de México. Ponencia presentada en el Encuentro Internacional de Intercambio de Experiencias Educativas 'Vincular los Caminos a la Educación Superior'”. Noviembre 17-19. Mexico, 2003.

The Task Force on Higher Education and Society (2000) Higher Education in Developing Countries. Peril and Promise. Washington, DC: The World Bank.

TORRES, C. A. (1998) Education, Democracy and Multiculturalism: Dilemmas of Citizenship in a Multicultural Society. Ladham, Md: Rowman and Littlefield.

UNESCO (1998) World Declaration on Higher Education for the Twenty-First Century: Vision and Action. Paris: UNESCO.

Universidad Intercultural del Estado de México (http://www.redui.org.mx) Agosto 29, 2007. 